\title{
Systematic review and audit of our series of intracapsular fracture fixation using a new implant; targon fn plate system with tips learned from our experience
}

\author{
Albert Tang*, Thisara C Weerasuriya and Francis Chan \\ Tameside General Hospital, UK
}

\section{Introduction}

The two most commonly used implants for the fixation of intracapsular fractures of the neck of the femur are the multiple parallel screw method and the sliding hip screw method. The sliding screw allowed for collapse of the bone at the fracture site and the multiple screw technique allowed for rotational stability. These two implants have individual mechanical properties which have been combined into the design of the TARGON plate.

\section{Methods}

The main objective of our study was to analyse the TARGON plate system used in a district general hospital to fix intra-capsular fractures with regard to operating time, metal work cut out, failure of fixation and avascular necrosis of the head of the femur.

The study was performed over a period of one year from 2012 to 2013 in three different district general hospitals in the UK. The plate system was only used to fix AO 31B1 and 31B2 fractures. All patients with the above fractures were included in the study. This was a retrospective study and involved three surgeons from different parts of the country.

The operative technique involved positioning the patient on the traction table and either fixing the fracture insitu or minimal manipulation to reduce the fracture and fixing. The approach was a direct lateral approach with an average incision length of $5 \mathrm{~cm}$. The fixation is performed using the jigs in the system.

The patients were followed up at 06 weeks in fracture clinic and 03 months. Long term follow-up is on-going.

\section{Results}

A total of 46 patients were identified who had the above fractures and were fixed with the TARGON plating system. Out of the 46 patients 33 were females and 13 were males. The age range was 71 years to 92 years. The mean age was 80 years. All were osteoporotic 31B1 and 31B2 fractures. All 46 were available for 6 week review while only 30 were followed up at 03 months. There were 08 deaths at 03 months. There were no deaths related to operative complications.

Nine patients had wound infection which were treated successfully

Copyright: $(02016$ Tang A. This is an open-access article distributed under the terms of the Creative Commons Attribution License, which permits unrestricted use, distribution, and reproduction in any medium, provided the original author and source are credited. with antibiotics. No implant removal was necessary. No deep infections were reported. Out of 46 patients only one patient had one of the screws cut out. This was revised to a bipolar hemi-arthroplasty. None of the patients had collapse of the femoral head due to avascular necrosis in this series.

The average operating time was 45 minutes and the range was 30-65 minutes.

\section{Discussion}

The two most commonly used implants for the fixation of intracapsular fractures of the neck of the femur are the multiple parallel screw method and the sliding hip screw method. The sliding screw allowed for collapse of the bone at the fracture site and the multiple screw technique allowed for rotational stability.

These two implants have been compared in their various features in six randomised trials using 772 participants. With sliding screws the incidence of fracture healing complications were lower (28\% versus $33 \%$ ). The sliding hip screw was associated with more wound healing complications probably due to the slightly longer time needed for the procedure.

The Targon is a design which incorporates the advantageous features of both implants. This device provides the rotational stability and the lateral support. The implant is Magnetic Resonance Imaging compatible.

The jig allowed the three screws which are telescopic, to be positioned in a triangular configuration providing rotational stability. Due to the jig the screw can be placed perfecrly parallel allowing collapse, which is further facilitated by the telescopic nature of the screws, the plate in the construct provided the lateral support.

We found that removing the handle of the jig helps to manoeuvre the jig more easily especially in the case obese patients.

\section{Conclusion}

We conclude from our limited experience that Targon device appears to be a promising implant in the treatment of intra-capsular fractures of the femur.

Correspondence to: Albert Tang, Tameside General Hospital, UK, E-mail: alberttang@doctors.org.uk

Received: May 07, 2016; Accepted: June 17, 2016; Published: June 20, 2016 